\title{
Análisis postural del trabajador forestal en aserraderos de El Salto, Durango, México
}

\section{Postural analysis of the forestry worker in sawmills of El Salto, Durango, Mexico}

\author{
Alondra Yareli Aragón-Vásquez', Edwin Daniel Silva-Lugo', Juan Abel Nájera-Lunal*, Jorge Méndez-González², \\ Francisco Javier Hernández' y Ricardo de la Cruz-Carreral
}

\begin{abstract}
1 Tecnológico Nacional de México. Instituto Tecnológico de El Salto. El Salto, Durango, México.

2 Universidad Autónoma Agraria Antonio Narro. * * Autor de correspondencia. jalnajera@itelsalto.edu.mx Departamento Forestal. Saltillo, Coahuila, México.
\end{abstract}

\section{RESUMEN}

El trabajo en la industria del aserrío es físicamente exigente porque se realiza bajo posturas corporales poco saludables que generan lesiones musculoesqueléticas y su estudio requiere de herramientas de evaluación de riesgos para identificarlas adecuadamente. En los aserraderos de El Salto, Durango, México se desconoce el nivel de riesgo de las posturas de trabajo en las personas, por lo que el objetivo de este estudio fue realizar un análisis postural en 15 puestos de trabajo. A partir de $7.5 \mathrm{~h}$ de video, que consideraron el trabajo efectivo en los puestos laborales, se obtuvieron 900 imágenes fijas a intervalos de 30 segundos para analizarlas mediante el método OWAS. Se estimó el índice global de riesgo postural y se elaboraron tablas de contingencia y pruebas de asociación entre variables categóricas de Chi-cuadrado. Los resultados mostraron una frecuencia de posturas de 48\% con nivel de riesgo 2, 3 y 4, indicando realizar acciones de corrección postural para los riesgos 2 y 3 así como modificaciones inmediatas para el 4. El índice global de riesgo se estableció en 166 puntos catalogado como mínimo. Por puesto de trabajo, los topadores y volteadores son los más susceptibles de presentar lesiones musculoesqueléticas con índices globales de riesgo por encima de los 200 puntos. Por zona corporal, $43 \%$ de las posiciones de la espalda y $40 \%$ de las piernas se encuentran en un nivel de riesgo 2, considerado como poco dañino, y $100 \%$ de las posiciones de los brazos resultaron con un nivel de riesgo 1 que no requiere modificación alguna.

PALABRAS CLAVE: ergonomía, factores de riesgo, índice global de riesgo postural, lesiones musculoesqueléticas, OWAS, puestos de trabajo.

\section{ABSTRACT}

The work in the sawmill industry is physically demanding because it is performed under unhealthy body postures that generate musculoskeletal injuries and its study requires risk assessment tools to identify the most serious. In the sawmills of El Salto, Durango, Mexico, the level of risk of the work positions in people is unknown; therefore, the objective of this study was to perform a postural analysis in 15 workplaces. From 7.5 hours of video that considered effective work in the workplace, 900 still images were obtained at 30second intervals to analyze them using the OWAS method. The global index of postural risk was estimated and contingency tables and association tests between categorical variables of chi square were elaborated. The results showed a frequency of postures of $48 \%$ with risk levels 2, 3 and 4, indicating actions for postural correction for risks 2 and 3 as well as immediate modifications for the 4. The global risk index was established at 166 points cataloged as minimum. By workplace, the workers in the manual logs handling and manual stacking and carriage of lumber are the most susceptible to present injuries with global risk indexes above 200 points. By body area, $43 \%$ of the positions of the back and $40 \%$ of the legs are in a level risk 2 considered as not harmful, and $100 \%$ of the positions of the arms resulted with a level risk 1 not requiring any modification.

KEYWORDS: ergonomics, risk factors, global index of postural risk, musculoskeletal injuries, OWAS, workplaces. 


\section{INTRODUCCIÓN}

El trabajo forestal se considera físicamente exigente en las operaciones donde los trabajadores a menudo deben realizar sus actividades bajo posturas corporales incómodas y poco saludables debido a los esfuerzos que representa levantar o transportar cargas pesadas y realizar movimientos repetitivos y peligrosos (Chandra et al., 2011). Los aserraderos tradicionales con frecuencia presentan un problema ergonómico, ya que se han estructurado para que el trabajador se adapte al diseño del puesto de trabajo en lugar de que el diseño se adapte al trabajador y, bajo este entorno de trabajo, es común que se presenten lesiones musculoesqueléticas que detonan impactos negativos en términos de eficiencia laboral, salud, seguridad y equilibrio psicológico de los empleados (Polat, Özkaya y Kalinkara, 2016).

Mejorar la productividad es el objetivo principal de cualquier empresa y la mejora continua entre el trabajador y su entorno es una de las alternativas más eficientes para mejorarla (Özkaya, Polat y Kalinkara, 2018). En este sentido, el cumplimiento de los objetivos de productividad de la industria del aserrío, considerando el equilibrio entre las características del trabajador y las demandas de las tareas realizadas, requiere darle importancia a la ergonomía de los puestos de trabajo (Qutubuddin, Hebbal y Kumar, 2013); sin embargo, la tendencia es emplear la ergonomía reactiva después de que las lesiones ya se han producido, en lugar de ser proactiva y prevenirlas (Pascual y Naqvi, 2008).

Uno de los factores de riesgo ocupacional más importantes que conducen a lesiones musculoesqueléticas en los trabajadores son las posturas deficientes e incómodas, si se considera que la postura de trabajo se define como la adecuada alineación de la cabeza, el cuerpo, los brazos y las piernas de acuerdo con el trabajo realizado (Unver-Okan, Acar y Kaya, 2017). Los factores de riesgo que hacen que las posturas de trabajo sean peligrosas se pueden enumerar como posiciones corporales fijas o restringidas, repetición continua de movimientos, un ritmo de trabajo que cause fatiga y que no permita una recuperación suficiente entre movimientos, así como el levantamiento y manipulación de objetos pesados (Gupta, 2006). El objetivo de un sistema de evaluación de riesgos es identificar los factores, sopesar su influencia relativa y proporcionar suficiente información para generar conciencia y medidas de mitigación. En consecuencia, el área general de la evaluación de riesgos es amplia, con muchos métodos y herramientas disponibles para diversos entornos (Liu, Martínez, Wang, Rodríguez y Novozhilov, 2010).

A este respecto, existen iniciativas de prevención de lesiones musculoesqueléticas que requieren el uso de herramientas de evaluación que identifican las posturas con mayor riesgo de lesiones; dichas herramientas de análisis cualitativo recopilan datos básicos de observación sobre un trabajo y generalmente requieren de un menor esfuerzo por parte del analista para su interpretación (Jones y Kumar, 2010). Entre los métodos utilizados para evaluar riesgos por posiciones forzadas del cuerpo humano, los indirectos son más económicos con respecto a los métodos directos, aunque requieren más tiempo de trabajo debido a la posterior interpretación de videos y/o fotografías (GómezGalán, Pérez-Alonso, Callejón-Ferre y López-Martínez, 2017).

Dentro de los métodos indirectos de análisis destaca el sistema de evaluación de la postura de trabajo de Ovako (OWAS, por sus siglas en inglés), el cual se basa en una clasificación simple y sistemática de las posturas de trabajo combinadas con observaciones de tareas de trabajo. Este método se puede aplicar a un puesto de trabajo para reducir su carga musculoesquelética y hacer que sea más seguro y productivo (Karhu, Kansi y Kuorinka, 1977). OWAS identifica diferentes tipos de posturas de la espalda más habituales en los trabajadores (4), brazos (3), piernas (7) y tres categorías de peso de cargas manejadas; esto implica hasta 252 combinaciones posibles, y a cada postura asumida con la categoría de carga respectiva se le asigna un nivel de riesgo que varía de 1 a 4 (Takala et al., 2010) y tiene la ventaja de ser bastante fácil de implementar, además, tiene un gran potencial para ser utilizado en la evaluación corporal de las operaciones forestales donde los trabajadores deben 
adoptar posturas incómodas cuando realizan sus tareas (Justavino, Ramírez, Pérez y Borz, 2015).

En los aserraderos de la región de El Salto, Pueblo Nuevo, Durango, no se tiene información sobre el riesgo que representa para los trabajadores adoptar determinadas posturas de trabajo durante la jornada laboral.

\section{OBjetivos}

Valorar el nivel de riesgo que representan la gama de posturas del trabajador forestal en 15 estaciones de trabajo que forman parte del proceso de producción de madera aserrada, susceptibles de generar lesiones musculoesqueléticas.

\section{MATERIALES Y MÉTODOS}

\section{Área de estudio}

El presente estudio se realizó en seis aserraderos ubicados en el municipio de Pueblo Nuevo del estado de Durango, México. El listado de los puestos de trabajo y las observaciones realizadas por aserradero se presentan en la tabla 1.
Los marcadores junto a los enganchadores conforman la tripulación del carro escuadra en el proceso de aserrío y su función es la de asegurar las trozas a las escuadras del carro y otorgar los gruesos de asierre a la madera en función de las indicaciones del aserrador, el cual es auxiliado por un ayudante que se encarga de mantener libre la salida de la sierra principal de materiales aserrados. Los volteadores se encuentran atentos a la indicación del aserrador para realizar los cambios de los planos de corte en las trozas aserradas, así como de alimentar el flujo de trocería al carro escuadra con el auxilio de ganchos madereros. Los operadores de maquinaria son el del montacargas, el de la reaserradora, el trocero y el de la desorilladora con sus respectivos ayudantes; cuando no se dispone de un sistema de desalojo y transporte automático de aserrín a las tolvas de almacenaje, se depende del aserrinero, quien se encarga de desalojarlo del foso de la sierra principal; esta tarea se hace completamente manual con auxilio de pala y carretilla. El clasificador se encarga de separar la madera aserrada por calidad y los topadores la distribuyen generalmente a hombro hacia las pilas separadas por calidad, grueso y largo.

TABLA 1. Observaciones por puestos de trabajo y aserradero.

\begin{tabular}{|c|c|c|c|c|c|c|c|}
\hline \multirow{2}{*}{ Puesto de trabajo } & \multicolumn{6}{|c|}{ Aserradero* } & \multirow{2}{*}{$\begin{array}{c}O B S \\
(n)\end{array}$} \\
\hline & $L V$ & $E D$ & $A G$ & $Q 1$ & $P N$ & $Q 2$ & \\
\hline Marcador & $\sqrt{ }$ & $\sqrt{ }$ & $\sqrt{ }$ & $\sqrt{ }$ & $\sqrt{ }$ & $\sqrt{ }$ & 60 \\
\hline Ayudante de desorilladora & $\sqrt{ }$ & $\sqrt{ }$ & & $\sqrt{ }$ & & $\sqrt{ }$ & 60 \\
\hline Aserrador & $\sqrt{ }$ & $\sqrt{ }$ & $\sqrt{ }$ & $\sqrt{ }$ & $\sqrt{ }$ & $\sqrt{ }$ & 60 \\
\hline Operador de montacargas & $\sqrt{ }$ & $\sqrt{ }$ & $\sqrt{ }$ & $\sqrt{ }$ & $\sqrt{ }$ & $\sqrt{ }$ & 60 \\
\hline Operador de reaserradora & $\sqrt{ }$ & & & & & & 60 \\
\hline Clasificador & $\sqrt{ }$ & & & $\sqrt{ }$ & & $\sqrt{ }$ & 60 \\
\hline Ayudante de reaserradora & $\sqrt{ }$ & & & & & & 60 \\
\hline Enganchador & $\sqrt{ }$ & $\sqrt{ }$ & $\sqrt{ }$ & $\sqrt{ }$ & $\sqrt{ }$ & $\sqrt{ }$ & 60 \\
\hline Ayudante de aserrador & $\sqrt{ }$ & $\sqrt{ }$ & $\sqrt{ }$ & $\sqrt{ }$ & $\sqrt{ }$ & $\sqrt{ }$ & 60 \\
\hline Ayudante de trocero & $\sqrt{ }$ & $\sqrt{ }$ & $\sqrt{ }$ & $\sqrt{ }$ & $\sqrt{ }$ & $\sqrt{ }$ & 60 \\
\hline Aserrinero & & $\sqrt{ }$ & $\sqrt{ }$ & $\sqrt{ }$ & $\sqrt{ }$ & $\sqrt{ }$ & 60 \\
\hline Operador de trocero & $\sqrt{ }$ & $\sqrt{ }$ & $\sqrt{ }$ & $\sqrt{ }$ & $\sqrt{ }$ & $\sqrt{ }$ & 60 \\
\hline Operador de desorilladora & $\sqrt{ }$ & $\sqrt{ }$ & & $\sqrt{ }$ & & $\sqrt{ }$ & 60 \\
\hline Volteadores & $\sqrt{ }$ & $\sqrt{ }$ & $\sqrt{ }$ & $\sqrt{ }$ & $\sqrt{ }$ & $\sqrt{ }$ & 60 \\
\hline Topadores & $\sqrt{ }$ & $\sqrt{ }$ & $\sqrt{ }$ & $\sqrt{ }$ & $\sqrt{ }$ & $\sqrt{ }$ & 60 \\
\hline
\end{tabular}

*LV=La Victoria; ED=El Diamante; AG=Aserradero García; Ql=Quintana I; PN=Pueblo Nuevo; Q2=Quintana II. OBS=Número de fotografias analizadas por puesto de trabajo. 


\section{Métodos}

\section{Colecta de información}

Durante el periodo de 16 de abril a 11 de junio 2018 se realizó la toma de información de campo. Para tal efecto, y con anterioridad, en cada aserradero se realizaron observaciones directas para identificar los ciclos y tareas de trabajo específicas de cada puesto laboral; posteriormente se procedió a registrarlos en video con una cámara digital de 16 megapíxeles de resolución. El conjunto de datos que se generó constó de 15 archivos de video (uno por cada puesto de trabajo con una duración mínima para cada uno de los seis aserraderos de 5 minutos que, al conjuntarlos, totalizaron 30 minutos por video) que consideraron solo el trabajo efectivo y de los cuales se obtuvo la captura de 60 tomas fotográficas de los trabajadores desempeñando sus funciones. En las tomas fotográficas se analizaron las posturas adoptadas dentro de los ciclos de trabajo como lo recomiendan Lee y Han (2013). En total, se obtuvo una muestra de 7.5 horas de video y 900 imágenes fijas realizadas en promedio cada $30 \mathrm{~s}$; es importante mencionar que el análisis de las fotografías fue realizado por un solo observador y que las demoras, los descansos y otras acciones no productivas se excluyeron del estudio.

El análisis de los videos y las fotografías se realizó mediante el uso del software libre KINOVEA ${ }^{\circledR}$ por la facilidad que presenta al medir con precisión los ángulos de las posturas adquiridas por los trabajadores en las tomas estáticas y calificarla mediante el sistema de puntuación del sistema de análisis de postura de trabajo Ovako OWAS (Louhevaara, Suurnäkki, Hinkkanen y Helminen, 1992) donde se asignan calificaciones de riesgo para cada una de las 252 posturas posibles que pueden ser adoptadas por los trabajadores.

Utilizando la aplicación interactiva del programa HSE.Ergo.OWAS app desarrollada por RNI Group (2018), cada fotografía se analizó y clasificó de acuerdo con la puntuación de cuatro niveles de riesgo que integra el método OWAS, donde el nivel de riesgo 1 corresponde a una postura normal y no requiere de intervención alguna; el riesgo 2 considera una postura ligeramente dañina y donde se deben tomar medidas correctivas durante la próxima revisión regular de los métodos de trabajo; el nivel de riesgo 3 señala que la postura adoptada por el trabajador es dañina y es necesario tomar medidas correctivas lo antes posible; finalmente, el nivel de riesgo 4 se encuentra referido a todas aquellas posturas extremadamente dañinas donde las medidas correctivas a considerar son inmediatas. Con esto fue posible determinar los riesgos posturales de cada puesto de trabajo.

Finalmente, se determinó el índice global de riesgo postural (I), el cual varía entre $100(100 \%$ de las observaciones con posturas en la clase 1) y 400 puntos (100\% de las observaciones con posturas en la clase 4) donde valores cercanos a los 400 puntos representan un mayor riesgo de lesiones musculoesqueléticas. Este índice se calcula mediante el promedio ponderado de las frecuencias de las puntuaciones de riesgo registradas para cada puesto de trabajo de acuerdo con la ecuación propuesta por Calvo (2009):

$$
I=[(a * 1)+(b * 2)+(c * 3)+(d * 4)] * 1
$$

Donde:

$I$ = Índice global de riesgo postural (adimensional)

$a, b, c, d=$ Frecuencia de las puntuaciones para cada nivel de riesgo registradas (n)

1,2,3,4 = Nivel de riesgo de cada categoría

\section{Análisis estadístico}

Con la calificación de cada postura asumida por los trabajadores en sus puestos laborales se elaboraron tablas cruzadas o de contingencia que incluyeron las frecuencias y porcentajes dentro de las categorías evaluadas. Se realizaron, además, pruebas no paramétricas de Chi cuadrado para evaluar el grado de asociación o independencia entre variables categóricas (Janicak, 2007). Para esto, se utilizaron tanto el método asintótico como la prueba exacta de Fisher, siempre que más de 20\% de las frecuencias esperadas tuvieran valores menores a cinco (Sharpe, 2015). La variable categórica que consideró el tipo de puesto laboral se relacionó con cada una de las 
posiciones de las zonas corporales de los trabajadores. Los datos se analizaron utilizando el paquete estadístico SPSS ver. 19 (IBM Corp., 2010).

\section{Resultados}

\section{Nivel de riesgo postural por puesto de trabajo}

Las frecuencias de las posturas corporales y el índice de riesgo para los diferentes puestos de trabajo indican que, aunque $51.56 \%$ de los operadores trabajan en posturas normales, hay algunas con mayor susceptibilidad de generar lesiones musculoesqueléticas, tal es el caso del puesto de topador, que presentó el mayor índice global de riesgo con 227 puntos (Tabla 2), debido a una mayor frecuencia de posiciones corporales $(55 \%)$ clasificadas con niveles de riesgo 3 y 4 con el código postural 2133.

El siguiente nivel de riesgo lo presentaron las personas que se desempeñan como volteadores con un índice global de riesgo postural de 202 puntos y un código postural de 2133 , en este puesto, $50 \%$ de las posturas corresponden al nivel de riesgo 3 debido al esfuerzo realizado al imprimir fuerza excesiva cuando se manipulan trozas de dimensiones y pesos muy considerables.

En ambos casos se requiere modificar las posturas corporales para evitar daños musculoesqueléticos. El resto de los puestos de trabajo no implican mayor gravedad en las distintas zonas corporales de los trabajadores en virtud de no superar el índice de riesgo global de 200 puntos (Tabla 2).

\section{Análisis postural por puesto de trabajo}

Los resultados indican que los puestos de trabajo de los aserraderos evaluados están altamente relacionados con las categorías de las posiciones corporales de los trabajadores $(\mathrm{p}<0.001)$. La estimación de las frecuencias sobre las

TABLA 2. Nivel de riesgo general por puesto de trabajo.

\begin{tabular}{lccccc}
\hline & \multicolumn{5}{c}{ Nivel de riesgo } \\
\multicolumn{1}{c}{ Puesto de trabajo } & \multicolumn{3}{c}{ (\%) } & Índice de riesgo \\
\cline { 2 - 5 } & 4 & 3 & 2 & 1 & \\
\hline Marcadores & 0.00 & 0.00 & 15.00 & 85.00 & 115 \\
Ayudantes de desorilladora & 0.00 & 1.67 & 30.00 & 68.33 & 133 \\
Aserradores & 0.00 & 3.33 & 30.00 & 66.67 & 137 \\
Operadores de montacargas & 0.00 & 0.00 & 43.33 & 56.67 & 143 \\
Operadores de reaserradora & 0.00 & 6.67 & 30.00 & 63.33 & 143 \\
Clasificadores & 0.00 & 3.33 & 40.00 & 56.67 & 147 \\
Ayudantes de reaserradora & 0.00 & 16.67 & 21.67 & 61.67 & 155 \\
Enganchadores & 0.00 & 8.33 & 48.33 & 43.33 & 165 \\
Ayudantes de aserrador & 0.00 & 18.33 & 31.67 & 50.00 & 168 \\
Ayudantes de trocero & 0.00 & 25.00 & 33.33 & 41.67 & 183 \\
Aserrineros & 0.00 & 15.00 & 53.33 & 31.67 & 183 \\
Operadores de trocero & 0.00 & 20.00 & 50.00 & 30.00 & 190 \\
Operadores de desorilladora & 0.00 & 30.00 & 33.33 & 36.67 & 193 \\
Volteadores & 0.00 & 50.00 & 1.67 & 48.33 & 202 \\
Topadores & 5.00 & 50.00 & 11.67 & 33.33 & 227 \\
\hline Promedio & 0.33 & 16.55 & 31.56 & 51.56 & 166 \\
\hline
\end{tabular}


posiciones corporales por puesto de trabajo señaladas en la tabla 3, permiten deducir por ejemplo, que los volteadores alternan la posición de la espalda en forma recta o inclinada con los brazos bajo el nivel de los hombros, pero es posible que el tipo de esfuerzo les exija levantar uno o los dos para aumentar la fuerza en combinación con palancas ejercidas por una o las dos piernas, ya que la exigencia de fuerza en este puesto es superior para manipular trozas sumamente pesadas; mientras que los topadores lo hacen pero con madera recién aserrada, asumiendo posiciones con la espalda en inclinaciones mayores a 20 grados con los brazos debajo de los hombros, elevándolos cuando sujetan la carga con uno o dos brazos por encima del nivel de los hombros realizando apoyos en una pierna o caminando hasta el patio de madera aserrada cargando un peso que rebasa en mucho los $20 \mathrm{~kg}$.

\section{Análisis postural de la espalda}

El análisis de la espalada reveló que 58\% de las posturas adquiridas por los trabajadores en los diferentes puestos laborales corresponde a un nivel de riesgo 1 que no tienen consecuencia alguna en términos de riesgos posturales, pero el restante $42 \%$ (exceptuando los puestos de los marcadores y los ayudantes de la desorilladora), se encuentran dentro de un nivel de riesgo 2 producto de posiciones inclinadas y giros de la espalda; aunque en general, el índice global de riesgo alcanzado de 142 puntos que sugiere un riesgo mínimo a la espalda (Tabla 4).

\section{Análisis postural de los brazos}

El análisis de la postura de los brazos mostró que su condición es segura pues $100 \%$ de las frecuencias observadas en cada puesto de trabajo se clasificaron en el nivel de riesgo 1, por lo que no es necesario realizar modificaciones (Tabla 5).

\section{Análisis postural de las piernas}

La mayoría de las posturas que realizan los trabajadores con las piernas $(60 \%)$, les corresponde un nivel de riesgo 1 y no ameritan modificación alguna; pero al restante $40 \%$ que incluye posiciones con una pierna recta y la otra inclinada para generar tracción, sentado o de pie por largos periodos, requieren modificación en sus posturas o incorporar periodos de descanso frecuentes para disminuir los periodos largos en una sola posición (Tabla 6).

\section{DISCUSIÓN}

Aunque existe poca información sobre la clasificación del índice global de riesgo que varía de 100 a 400 puntos, se considera que valores superiores a 250 puntos representan un alto riesgo y menores a ese valor representan un riesgo moderado para el trabajador (Zanuttini, Cielo y Poncino, 2005). En función a lo anterior, la puntuación promedio alcanzada en este estudio de 166 puntos se puede considerar como un nivel de riesgo mínimo en el que se requerirán acciones correctivas a corto plazo; mientras que, por puesto de trabajo, los 227 puntos alcanzados por los topadores y 202 por los volteadores, reflejan una situación de riesgo moderado donde se requiere adoptar posiciones corporales más apropiadas para minimizar riesgos musculoesqueléticos. Es probable que los trabajadores de estos puestos ya presenten afectaciones debido al peso y longitud de las tablas que tienen que soportar al transportarlas desde la línea de clasificación hasta el patio de madera aserrada, superando en muchos casos el límite de cargas de $50 \mathrm{~kg}$ y cuatro metros de longitud recomendadas por la NOM-006-STPS-2014 de la Secretaría del Trabajo y Previsión Social [STPS], (2014).

Cuando se detecta un nivel de riesgo elevado en trabajadores expuestos a posturas forzadas, se debe implementar un protocolo médico específico de vigilancia para realizar exámenes del sistema osteomuscular y valorar las dolencias a partir de palpaciones y movilidad de miembros y articulaciones con la finalidad de emitir un diagnóstico y en consecuencia, un programa de rehabilitación o recuperación del trabajador mediante la modificación de las posturas riesgosas, rotaciones entre puestos de trabajo, pausas de recuperación, tratamientos médicos o intervenciones quirúrgicas (CISNS, 2000). 
TABLA 3. Análisis postural del cuerpo por puesto de trabajo.

Puestos de trabajo

\begin{tabular}{|c|c|c|c|c|c|c|c|c|c|c|c|c|c|c|c|c|}
\hline \multirow[b]{2}{*}{$\begin{array}{l}\text { Posición de la zona } \\
\text { corporal }\end{array}$} & \multicolumn{15}{|c|}{ Puestos de trabajo } & \multirow[b]{2}{*}{$\begin{array}{l}\text { Valor de } \\
\qquad p^{*}\end{array}$} \\
\hline & 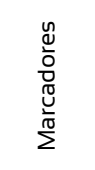 & 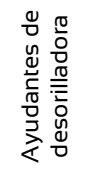 & 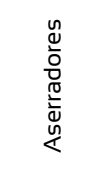 & 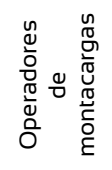 & 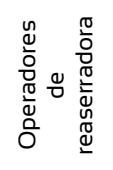 & 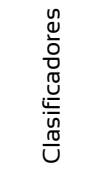 & 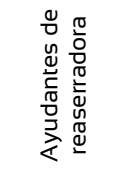 & 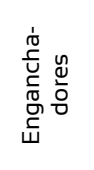 & 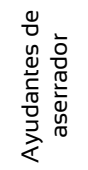 & 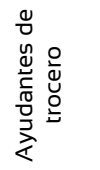 & 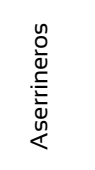 & 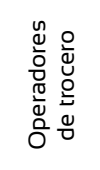 & 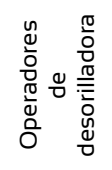 & 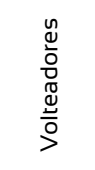 & 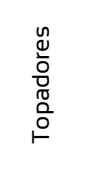 & \\
\hline \multicolumn{17}{|l|}{ Espalda } \\
\hline Recta (n) & $\begin{array}{c}41 \\
(68.3)\end{array}$ & $\begin{array}{c}32 \\
(53.3)\end{array}$ & $\begin{array}{c}31 \\
(51.7)\end{array}$ & $\begin{array}{c}27 \\
(45.0)\end{array}$ & $\begin{array}{c}39 \\
(65.0)\end{array}$ & $\begin{array}{c}34 \\
(56.7)\end{array}$ & $\begin{array}{c}36 \\
(60.0)\end{array}$ & $\begin{array}{c}23 \\
(38.3)\end{array}$ & $\begin{array}{c}22 \\
(36.7)\end{array}$ & $\begin{array}{c}19 \\
(31.7)\end{array}$ & $\begin{array}{c}9 \\
(15.0)\end{array}$ & $\begin{array}{c}16 \\
(26.7)\end{array}$ & $\begin{array}{c}20 \\
(33.3)\end{array}$ & $\begin{array}{c}29 \\
(48.3)\end{array}$ & $\begin{array}{c}22 \\
(36.7)\end{array}$ & \\
\hline Inclinada (n) & $\begin{array}{c}9 \\
(15.0)\end{array}$ & $\begin{array}{c}18 \\
(30.0)\end{array}$ & $\begin{array}{c}19 \\
(31.7)\end{array}$ & $\begin{array}{c}26 \\
(43.3)\end{array}$ & $\underset{(33.3)}{20}$ & $\begin{array}{c}26 \\
(43.3)\end{array}$ & $\begin{array}{c}23 \\
(38.3)\end{array}$ & $\begin{array}{c}33 \\
(55.0)\end{array}$ & $\begin{array}{c}28 \\
(46.7)\end{array}$ & $\begin{array}{c}34 \\
(56.7)\end{array}$ & $\begin{array}{c}37 \\
(61.7)\end{array}$ & $\begin{array}{c}39 \\
(65.0)\end{array}$ & $\begin{array}{c}36 \\
(60.0)\end{array}$ & $\begin{array}{c}30 \\
(50.0)\end{array}$ & $\begin{array}{c}31 \\
(51.7)\end{array}$ & * נחתחת \\
\hline Con giro (n) & $\stackrel{9}{(15.0)}$ & $\stackrel{9}{(15.0)}$ & $\begin{array}{c}9 \\
(15.0)\end{array}$ & $\begin{array}{c}7 \\
(11.7)\end{array}$ & $\begin{array}{c}1 \\
(1.7)\end{array}$ & $\begin{array}{c}0 \\
(0.0)\end{array}$ & $\begin{array}{c}1 \\
(1.7)\end{array}$ & $\begin{array}{c}3 \\
(5.0)\end{array}$ & $\begin{array}{c}9 \\
(15.0)\end{array}$ & $\begin{array}{c}6 \\
(10.0)\end{array}$ & $\begin{array}{c}12 \\
(20.0)\end{array}$ & $\begin{array}{c}2 \\
(3.3)\end{array}$ & $\begin{array}{c}4 \\
(6.7)\end{array}$ & $\begin{array}{c}1 \\
(1.7)\end{array}$ & $\begin{array}{c}2 \\
(3.3)\end{array}$ & 0.0001 \\
\hline $\begin{array}{l}\text { Inclinada con giro } \\
\text { (n) }\end{array}$ & $\begin{array}{c}1 \\
(1.7)\end{array}$ & $\begin{array}{c}1 \\
(1.7)\end{array}$ & $\begin{array}{c}1 \\
(1.7)\end{array}$ & $\begin{array}{c}0 \\
(0.0)\end{array}$ & $\begin{array}{c}0 \\
(0.0)\end{array}$ & $\begin{array}{c}0 \\
(0.0)\end{array}$ & $\begin{array}{c}0 \\
(0.0)\end{array}$ & $\begin{array}{c}1 \\
(1.7)\end{array}$ & $\begin{array}{c}1 \\
(1.7)\end{array}$ & $\begin{array}{c}1 \\
(1.7)\end{array}$ & $\stackrel{2}{2}$ & $\begin{array}{c}3 \\
(5.0)\end{array}$ & $\begin{array}{c}0 \\
(0.0)\end{array}$ & $\begin{array}{c}0 \\
(0.0)\end{array}$ & $\begin{array}{c}5 \\
(8.3)\end{array}$ & \\
\hline \multicolumn{17}{|l|}{ Brazos } \\
\hline $\begin{array}{l}\text { Los dos debajo de } \\
\text { los hombros (n) }\end{array}$ & $\begin{array}{c}59 \\
(98.3)\end{array}$ & $\begin{array}{c}58 \\
(96.7)\end{array}$ & $\begin{array}{c}58 \\
(96.7)\end{array}$ & $\begin{array}{c}60 \\
(100.0)\end{array}$ & $\begin{array}{c}59 \\
(98.3)\end{array}$ & $\begin{array}{c}60 \\
(100.0)\end{array}$ & $\begin{array}{c}60 \\
(100.0)\end{array}$ & $\begin{array}{c}55 \\
(91.7)\end{array}$ & $\begin{array}{c}58 \\
(96.7)\end{array}$ & $\begin{array}{c}60 \\
(100.0)\end{array}$ & $\begin{array}{c}59 \\
(98.3)\end{array}$ & $\begin{array}{c}60 \\
(100.0)\end{array}$ & $\begin{array}{c}60 \\
(100.0)\end{array}$ & $\begin{array}{c}39 \\
(65.0)\end{array}$ & $\begin{array}{c}40 \\
(66.7)\end{array}$ & \\
\hline Uno elevado (n) & $\begin{array}{c}1 \\
(1.7)\end{array}$ & $\begin{array}{c}2 \\
(3.3)\end{array}$ & $\begin{array}{c}2 \\
(3.3)\end{array}$ & $\begin{array}{c}0 \\
(0.0)\end{array}$ & $\begin{array}{c}0 \\
(0.0)\end{array}$ & $\begin{array}{c}0 \\
(0.0)\end{array}$ & $\begin{array}{r}0 \\
(0.0)\end{array}$ & $\begin{array}{c}1 \\
(1.7)\end{array}$ & $\begin{array}{c}2 \\
(3.3)\end{array}$ & $\begin{array}{c}0 \\
(0.0)\end{array}$ & $\begin{array}{c}1 \\
(1.7)\end{array}$ & $\begin{array}{c}0 \\
(0.0)\end{array}$ & $\begin{array}{c}0 \\
(0.0)\end{array}$ & $\begin{array}{c}10 \\
(16.7)\end{array}$ & $\begin{array}{c}10 \\
(16.7)\end{array}$ & $0.0001^{*}$ \\
\hline $\begin{array}{l}\text { Los dos elevados } \\
\text { (n) }\end{array}$ & $\begin{array}{c}0 \\
(0.0) \\
\end{array}$ & $\begin{array}{c}0 \\
(0.0) \\
\end{array}$ & $\begin{array}{c}0 \\
(0.0) \\
\end{array}$ & $\begin{array}{c}0 \\
(0.0) \\
\end{array}$ & $\begin{array}{c}1 \\
(1.7)\end{array}$ & $\begin{array}{c}0 \\
(0.0) \\
\end{array}$ & $\begin{array}{c}0 \\
(0.0) \\
\end{array}$ & $\begin{array}{c}4 \\
(6.7) \\
\end{array}$ & $\begin{array}{c}0 \\
(0.0) \\
\end{array}$ & $\begin{array}{c}0 \\
(0.0) \\
\end{array}$ & $\begin{array}{c}0 \\
(0.0) \\
\end{array}$ & $\begin{array}{c}0 \\
(0.0)\end{array}$ & $\begin{array}{c}0 \\
(0.0)\end{array}$ & $\begin{array}{c}11 \\
(18.3) \\
\end{array}$ & $\begin{array}{c}10 \\
(16.7)\end{array}$ & \\
\hline \multicolumn{17}{|l|}{ Piernas } \\
\hline Sentado (n) & $\begin{array}{c}36 \\
(60.0)\end{array}$ & $\begin{array}{c}0 \\
(0.0)\end{array}$ & $\begin{array}{c}23 \\
(38.3)\end{array}$ & $\begin{array}{c}60 \\
(100.0)\end{array}$ & $\begin{array}{c}0 \\
0\end{array}$ & $\begin{array}{c}0 \\
(0.0)\end{array}$ & $\begin{array}{c}0 \\
0\end{array}$ & $\begin{array}{c}5 \\
(8.3)\end{array}$ & $\begin{array}{c}0 \\
(0.0)\end{array}$ & $\begin{array}{c}0 \\
(0.0)\end{array}$ & $\begin{array}{c}0 \\
(0.0)\end{array}$ & $\begin{array}{c}0 \\
(0.0)\end{array}$ & $\begin{array}{c}0 \\
(0.0)\end{array}$ & $\begin{array}{c}0 \\
(0.0)\end{array}$ & $\begin{array}{c}0 \\
0\end{array}$ & \\
\hline De pie (n) & $\begin{array}{c}20 \\
(33.3)\end{array}$ & $\begin{array}{c}27 \\
(45.0)\end{array}$ & $\begin{array}{c}32 \\
(53.3)\end{array}$ & $\begin{array}{c}0 \\
(0.0)\end{array}$ & $\begin{array}{c}54 \\
(90.0)\end{array}$ & $\begin{array}{c}47 \\
(78.3)\end{array}$ & $\begin{array}{c}53 \\
(88.3)\end{array}$ & $\begin{array}{c}34 \\
(56.7)\end{array}$ & $\begin{array}{c}25 \\
(41.6)\end{array}$ & $\begin{array}{c}49 \\
(81.7)\end{array}$ & $\begin{array}{c}19 \\
(31.7)\end{array}$ & $\begin{array}{c}37 \\
(61.7)\end{array}$ & $\begin{array}{c}33 \\
(55.0)\end{array}$ & $\begin{array}{c}19 \\
(31.7)\end{array}$ & $\begin{array}{c}18 \\
(30.0)\end{array}$ & \\
\hline $\begin{array}{l}\text { Apoyo en una } \\
\text { pierna (n) }\end{array}$ & $\begin{array}{c}4 \\
(6.7)\end{array}$ & $\begin{array}{c}33 \\
(55.0)\end{array}$ & $\begin{array}{c}3 \\
(5.0)\end{array}$ & $\begin{array}{c}0 \\
(0.0)\end{array}$ & $\begin{array}{c}6 \\
(10.0)\end{array}$ & $\begin{array}{c}4 \\
(3.7)\end{array}$ & $\begin{array}{c}7 \\
\text { (11.7) }\end{array}$ & $\begin{array}{c}18 \\
(30.0)\end{array}$ & $\begin{array}{c}34 \\
(56.7)\end{array}$ & $\begin{array}{c}11 \\
(18.3)\end{array}$ & $\begin{array}{c}36 \\
(60.0)\end{array}$ & $\begin{array}{c}22 \\
(36.7)\end{array}$ & $\begin{array}{c}27 \\
(45.0)\end{array}$ & $\begin{array}{c}40 \\
(66.7)\end{array}$ & $\begin{array}{c}31 \\
(51.7)\end{array}$ & \\
\hline $\begin{array}{l}\text { Rodillas } \\
\text { flexionadas } \\
\text { equilibrado (n) }\end{array}$ & $\begin{array}{c}0 \\
(0.0)\end{array}$ & $\begin{array}{c}0 \\
(0.0)\end{array}$ & $\begin{array}{c}2 \\
(3.3)\end{array}$ & $\begin{array}{c}0 \\
(0.0)\end{array}$ & $\begin{array}{c}0 \\
(0.0)\end{array}$ & $\begin{array}{c}0 \\
(0.0)\end{array}$ & $\begin{array}{c}0 \\
(0.0)\end{array}$ & $\begin{array}{c}3 \\
(5.0)\end{array}$ & $\begin{array}{c}0 \\
(0.0)\end{array}$ & $\begin{array}{c}0 \\
(0.0)\end{array}$ & $\begin{array}{c}4 \\
(6.7)\end{array}$ & $\begin{array}{c}0 \\
(0.0)\end{array}$ & $\begin{array}{c}0 \\
(0.0)\end{array}$ & $\begin{array}{c}1 \\
(1.7)\end{array}$ & $\begin{array}{c}1 \\
(1.7)\end{array}$ & $0.0001^{*}$ \\
\hline $\begin{array}{l}\text { Rodillas } \\
\text { flexionadas } \\
\text { desequilibrado (n) }\end{array}$ & $\begin{array}{c}0 \\
(0.0)\end{array}$ & $\begin{array}{c}0 \\
(0.0)\end{array}$ & $\begin{array}{c}0 \\
(0.0)\end{array}$ & $\begin{array}{c}0 \\
(0.0)\end{array}$ & $\begin{array}{c}0 \\
(0.0)\end{array}$ & $\begin{array}{c}0 \\
(0.0)\end{array}$ & $\begin{array}{c}0 \\
(0.0)\end{array}$ & $\begin{array}{c}0 \\
(0.0)\end{array}$ & $\begin{array}{c}0 \\
(0.0)\end{array}$ & $\begin{array}{c}0 \\
(0.0)\end{array}$ & $\begin{array}{c}0 \\
(0.0)\end{array}$ & $\begin{array}{c}0 \\
(0.0)\end{array}$ & $\begin{array}{c}0 \\
(0.0)\end{array}$ & $\begin{array}{c}0 \\
(0.0)\end{array}$ & $\begin{array}{c}0 \\
(0.0)\end{array}$ & \\
\hline Arrodillado (n) & $\begin{array}{c}0 \\
(0.0)\end{array}$ & $\begin{array}{c}0 \\
(0.0)\end{array}$ & $\begin{array}{c}0 \\
(0.0)\end{array}$ & $\begin{array}{c}0 \\
(0.0)\end{array}$ & $\begin{array}{c}0 \\
(0.0)\end{array}$ & $\begin{array}{c}0 \\
(0.0)\end{array}$ & $\begin{array}{c}0 \\
(0.0)\end{array}$ & $\begin{array}{c}0 \\
(0.0)\end{array}$ & $\begin{array}{c}0 \\
(0.0)\end{array}$ & $\begin{array}{c}0 \\
(0.0)\end{array}$ & $\begin{array}{c}0 \\
(0.0)\end{array}$ & $\begin{array}{c}0 \\
(0.0)\end{array}$ & $\begin{array}{c}0 \\
(0.0)\end{array}$ & $\begin{array}{c}0 \\
(0.0)\end{array}$ & $\begin{array}{c}0 \\
(0.0)\end{array}$ & \\
\hline Caminando (n) & $\begin{array}{c}0 \\
(0.0) \\
\end{array}$ & $\begin{array}{c}0 \\
(0.0) \\
\end{array}$ & $\begin{array}{c}0 \\
(0.0) \\
\end{array}$ & $\begin{array}{c}0 \\
(0.0) \\
\end{array}$ & $\begin{array}{c}0 \\
(0.0) \\
\end{array}$ & $\begin{array}{c}9 \\
(15.0)\end{array}$ & $\begin{array}{c}0 \\
(0.0) \\
\end{array}$ & $\begin{array}{c}0 \\
(0.0) \\
\end{array}$ & $\begin{array}{c}1 \\
(1.7)\end{array}$ & $\begin{array}{c}0 \\
(0.0) \\
\end{array}$ & $\begin{array}{c}1 \\
(1.7)\end{array}$ & $\begin{array}{c}1 \\
(1.7)\end{array}$ & $\begin{array}{c}0 \\
(0.0) \\
\end{array}$ & $\begin{array}{c}0 \\
(0.0)\end{array}$ & $\begin{array}{c}10 \\
(16.7) \\
\end{array}$ & \\
\hline \multicolumn{17}{|l|}{ Carga o esfuerzo } \\
\hline Menos de 10 kg (n) & $\begin{array}{c}56 \\
(93.3)\end{array}$ & $\begin{array}{c}42 \\
(70.0)\end{array}$ & $\begin{array}{c}60 \\
(100.0)\end{array}$ & $\begin{array}{c}60 \\
(100.0)\end{array}$ & $\begin{array}{c}39 \\
(65.0)\end{array}$ & $\begin{array}{c}40 \\
(66.7)\end{array}$ & $\begin{array}{c}32 \\
(53.3)\end{array}$ & $\begin{array}{c}23 \\
(38.3)\end{array}$ & $\begin{array}{c}32 \\
(53.3)\end{array}$ & $\begin{array}{c}24 \\
(35.0)\end{array}$ & $\begin{array}{c}41 \\
(68.3)\end{array}$ & $\begin{array}{c}15 \\
(25.0)\end{array}$ & $\begin{array}{c}8 \\
(13.3)\end{array}$ & $\begin{array}{c}19 \\
(31.7)\end{array}$ & $\begin{array}{c}3 \\
(5.0)\end{array}$ & \\
\hline Entre 10 y 20 kg (n) & $\begin{array}{c}4 \\
(6.7)\end{array}$ & $\begin{array}{c}17 \\
(28.3)\end{array}$ & $\begin{array}{c}0 \\
(0.0)\end{array}$ & $\begin{array}{c}0 \\
(0.0)\end{array}$ & $\begin{array}{c}7 \\
\text { (11.7) }\end{array}$ & $\begin{array}{c}18 \\
(30.0)\end{array}$ & $\begin{array}{c}18 \\
(30.0)\end{array}$ & $\begin{array}{c}35 \\
(58.3)\end{array}$ & $\begin{array}{c}18 \\
(30.0)\end{array}$ & $\begin{array}{c}15 \\
(25.0)\end{array}$ & $\begin{array}{c}10 \\
(16.7)\end{array}$ & $\begin{array}{c}32 \\
(53.3)\end{array}$ & $\begin{array}{c}20 \\
(33.3)\end{array}$ & $\begin{array}{c}0 \\
(0.0)\end{array}$ & $\begin{array}{c}2 \\
(3.3)\end{array}$ & $0.0001^{*}$ \\
\hline Más de 20 kg (n) & $\begin{array}{c}0 \\
(0.0) \\
\end{array}$ & $\begin{array}{c}1 \\
(1.7) \\
\end{array}$ & $\begin{array}{c}0 \\
(0.0) \\
\end{array}$ & $\begin{array}{c}0 \\
(0.0) \\
\end{array}$ & $\begin{array}{c}14 \\
(23.3) \\
\end{array}$ & $\begin{array}{c}2 \\
(3.3)\end{array}$ & $\begin{array}{c}10 \\
(16.7) \\
\end{array}$ & $\begin{array}{c}2 \\
(3.3) \\
\end{array}$ & $\begin{array}{c}10 \\
(16.7) \\
\end{array}$ & $\begin{array}{c}21 \\
(35.0)\end{array}$ & $\begin{array}{c}9 \\
(15.0)\end{array}$ & $\begin{array}{c}13 \\
(21.7) \\
\end{array}$ & $\begin{array}{c}32 \\
(53.3) \\
\end{array}$ & $\begin{array}{c}41 \\
(68.3) \\
\end{array}$ & $\begin{array}{c}55 \\
(91.7) \\
\end{array}$ & \\
\hline
\end{tabular}

a Valor esperado de la prueba de $\mathrm{Chi}^{2}$, significativo $(\mathrm{p}<\mathrm{0.05}) \mathrm{n}$ = frecuencia. Entre paréntesis se presenta la frecuencia relativa (\%). 
TABLA 4. Índice de riesgo global para la espalda por puesto de trabajo.

\begin{tabular}{|c|c|c|c|c|c|}
\hline \multirow{2}{*}{ Puesto de trabajo } & \multicolumn{4}{|c|}{$\begin{array}{c}\text { Nivel de riesgo para la espalda } \\
(\%)\end{array}$} & \multirow{2}{*}{ Índice de riesgo } \\
\hline & 4 & 3 & 2 & 1 & \\
\hline Marcadores & 0.00 & 0.00 & 0.00 & 100.00 & 100 \\
\hline Ayudante de desorilladora & 0.00 & 0.00 & 0.00 & 100.00 & 100 \\
\hline Aserrador & 0.00 & 0.00 & 31.70 & 68.30 & 132 \\
\hline Operador de reaserradora & 0.00 & 0.00 & 33.30 & 66.70 & 133 \\
\hline Ayudante de reaserradora & 0.00 & 0.00 & 38.30 & 61.70 & 138 \\
\hline Operador de montacargas & 0.00 & 0.00 & 43.30 & 56.70 & 143 \\
\hline Clasificadores & 0.00 & 0.00 & 43.30 & 56.70 & 143 \\
\hline Ayudante de aserrador & 0.00 & 0.00 & 46.70 & 53.30 & 147 \\
\hline Volteador & 0.00 & 0.00 & 50.00 & 50.00 & 150 \\
\hline Topadores & 0.00 & 0.00 & 51.70 & 48.30 & 152 \\
\hline Enganchadores & 0.00 & 0.00 & 55.00 & 45.00 & 155 \\
\hline Ayudante de trocero & 0.00 & 0.00 & 56.70 & 43.30 & 157 \\
\hline Operador de desorilladora & 0.00 & 0.00 & 60.00 & 40.00 & 160 \\
\hline Aserrineros & 0.00 & 0.00 & 61.70 & 38.30 & 162 \\
\hline Operador de trocero & 0.00 & 0.00 & 65.00 & 35.00 & 165 \\
\hline Promedio & 0.00 & 0.00 & 42.45 & 57.55 & 142 \\
\hline
\end{tabular}

TABLA 5. Índice de riesgo global para los brazos por puesto de trabajo.

\begin{tabular}{lccccc}
\hline \multirow{2}{*}{\multicolumn{1}{c}{ Puesto de trabajo }} & \multicolumn{3}{c}{ Nivel de riesgo para los brazos (\%) } & \multirow{2}{*}{ Índice de riesgo } \\
\cline { 2 - 5 } & 4 & 3 & 2 & 1 & 100 \\
\hline Marcadores & 0.00 & 0.00 & 0.00 & 100.00 & 100 \\
Ayudante de desorilladora & 0.00 & 0.00 & 0.00 & 100.00 & 100 \\
Aserrador & 0.00 & 0.00 & 0.00 & 100.00 & 100 \\
Operador de montacargas & 0.00 & 0.00 & 0.00 & 100.00 & 100 \\
Operador de reaserradora & 0.00 & 0.00 & 0.00 & 100.00 & 100 \\
Clasificadores & 0.00 & 0.00 & 0.00 & 100.00 & 100 \\
Ayudante de reaserradora & 0.00 & 0.00 & 0.00 & 100.00 & 100 \\
Enganchadores & 0.00 & 0.00 & 0.00 & 100.00 & 100 \\
Ayudante de aserrador & 0.00 & 0.00 & 0.00 & 100.00 & 100 \\
Ayudante de trocero & 0.00 & 0.00 & 0.00 & 100.00 & 100 \\
Aserrineros & 0.00 & 0.00 & 0.00 & 100.00 & 100 \\
Operador de trocero & 0.00 & 0.00 & 0.00 & 100.00 & 100 \\
Operador de desorilladora & 0.00 & 0.00 & 0.00 & 100.00 & 100 \\
Volteador & 0.00 & 0.00 & 0.00 & 100.00 & 100 \\
Topadores & 0.00 & 0.00 & 0.00 & 100.00 & 100 \\
\hline Promedio & 0.00 & 0.00 & 0.00 & 100.00 & \\
\hline
\end{tabular}


TABLA 6. Índice de riesgo global para las piernas por puesto de trabajo.

\begin{tabular}{lccccc}
\hline & \multicolumn{3}{c}{ Nivel de riesgo para las piernas } & \multirow{2}{*}{ (\%) } \\
\cline { 2 - 5 } \multicolumn{1}{c}{ Puesto de trabajo } & 4 & 3 & 2 & 1 & \\
\hline Marcadores & 0.00 & 0.00 & 0.00 & 100.00 & 100 \\
Aserrador & 0.00 & 0.00 & 0.00 & 100.00 & 100 \\
Clasificadores & 0.00 & 0.00 & 0.00 & 100.00 & 100 \\
Enganchadores & 0.00 & 0.00 & 0.00 & 100.00 & 100 \\
Ayudante de aserrador & 0.00 & 0.00 & 0.00 & 100.00 & 100 \\
Ayudante de trocero & 0.00 & 0.00 & 0.00 & 100.00 & 100 \\
Operador de trocero & 0.00 & 0.00 & 36.70 & 63.30 & 137 \\
Operador de desorilladora & 0.00 & 0.00 & 45.00 & 55.00 & 145 \\
Topadores & 0.00 & 0.00 & 51.70 & 48.30 & 152 \\
Ayudante de desorilladora & 0.00 & 0.00 & 55.00 & 45.00 & 155 \\
Aserrineros & 0.00 & 0.00 & 60.00 & 40.00 & 160 \\
Volteador & 0.00 & 0.00 & 66.70 & 33.30 & 167 \\
Ayudante de reaserradora & 0.00 & 0.00 & 88.30 & 11.70 & 188 \\
Operador de reaserradora & 0.00 & 0.00 & 90.00 & 10.00 & 190 \\
Operador de montacargas & 0.00 & 0.00 & 100.00 & 0.00 & 200 \\
\hline Promedio & 0.00 & 0.00 & 39.56 & 60.44 & 140 \\
\hline
\end{tabular}

En operaciones forestales de abastecimiento, Calvo (2009) encontró que, en las fases del derribo y extracción manual de trozas, los valores del índice global de riesgos oscilan en los 300 puntos: la fase de derribo alcanzó valores de 287 puntos; la extracción manual 311 y el apilado manual 300 puntos, lo cual clasifica a estas actividades con un índice global de riesgo alto. Cheța, Marcu y Borz (2018), encontraron que el índice global de riesgo postural durante el derribo de árboles fue de 275 puntos, lo que ubica a esta actividad entre los índices de riesgo postural típicos encontrados para el trabajo forestal donde más de $50 \%$ de las frecuencias posturales se ubican dentro de los niveles de riesgo 3 y 4 que corresponden a posiciones con la espalda inclinada o torcida y las rodillas dobladas.

En la operación de descortezado con alimentación manual, Spinelli, Aminti, Magagnotti y De Francesco (2018), registraron un índice de riesgo de 136 puntos, mientras que para la carga y descarga manual de las trozas en la descortezadora arrojaron un índice de 146 y 129 puntos respectivamente. El riesgo postural incurrido durante el descortezado se considera relativamente bajo y equiparable con los índices de menor riesgo en los puestos de trabajo obtenidos en este estudio.

En general, en la mayoría de los puestos de trabajo de la industria del aserrío de la región de El Salto, Durango, se observó que las posturas inadecuadas catalogadas con un nivel de riesgo 2 y 3 se asocian al manejo y levantamiento de cargas que requieren de un considerable esfuerzo por parte del trabajador y que contribuyen a que se presenten lesiones lumbares por la constante exigencia de movimientos de inclinación de más de 20 grados de la espalda en combinación con la masa de los objetos a movilizar (Padula y Coury, 2003).

Eroglu, Kayacan y Yilmaz (2015) mencionan que el trabajo forestal ha sido definido fisiológicamente como una profesión de servicio pesado, y aunque en los últimos años 
ha habido en el sector un aumento en el uso de maquinaria, los operarios en los aserraderos siguen siendo sujetos a utilizar los músculos de sus piernas, manos, brazos, cabeza, cuello y hombros; lo cual quedó comprobado en este estudio (Tabla 3).

Es importante destacar también que solo el aserradero La Victoria cuenta con mecanismos de cadena transportadora de materiales que facilita su movilización, en aparente ventaja con los demás aserraderos bajo estudio en donde los trabajadores a menudo se convierten en transportadores que empujan, ruedan o cargan los materiales y subproductos del proceso, lo que explica de alguna manera su situación postural y que seguramente sea una de las causa principales de riesgo en contraer lesiones musculoesqueléticas en el presente o el futuro cercano; lo anterior se agrava porque los trabajadores no son dados a utilizar equipo de protección personal como fajas o dispositivos de seguridad que les protejan de una mejor forma las partes del cuerpo más expuesta a lesiones y daños corporales.

Bello y Mijinyawa (2010), encontraron en trabajadores de aserraderos del sur occidente de Nigeria una tasa de 58\% de lesiones y deformidades en la espalda y la espalda baja como resultado de las posiciones forzadas derivadas de la manipulación de trozas y madera aserrada pesadas; mencionan que, debido a que esas lesiones tardan mucho tiempo en manifestarse, cuando lo hacen, los trabajadores no las consideran una lesión musculoesquelética sino una deformación natural. Hoogendoorn et al. (2002) mencionan que la flexión y rotación de la espalda en más de 30 grados así como mantener esa postura durante más de 15 minutos aumenta considerablemente el riesgo de lesiones musculoesqueléticas y lumbares, por lo que es importante reducir las posiciones críticas el menor tiempo posible ya que se ha encontrado una relación inversa entre el aumento de la carga de trabajo y la postura óptima en trabajadores forestales, pues un aumento en la carga de trabajo resulta en la degeneración de los huesos y músculos debido a una postura deteriorada de la espalda (Quemelo, Gasparato y Vieira, 2015).
Aunque el riesgo postural de los brazos es bajo, una de las desventajas del método OWAS es que no evalúa más allá de que se encuentren por encima o debajo del nivel de los hombros; en este estudio se observó un uso excesivo de las manos y las muñecas en movimientos repetitivos en la mayoría de los puestos de trabajo y según Chandra, Ghosh, Asahara, Barman, Dev y Gangopadhyay (2011) esta constante repetición de tareas conducen a indicios de incomodidad en el cuello, los hombros y las muñecas de los trabajadores porque los músculos de la columna cervical y las articulaciones de los hombros pueden ejercer mayores fuerzas para controlar los movimientos de los brazos, lo que puede generar dolor, cansancio e incomodidad, ya que mientras las extremidades superiores están cargadas con tareas repetitivas, simultáneamente la espalda y las extremidades inferiores se cargan al mismo tiempo (Roman-Liu, 2014), por lo que la combinación de posturas forzadas, repetición de tareas, excesiva fuerza de agarre y las vibraciones de la maquinaria son factores de riesgo para que se presenten trastornos musculoesqueléticos en las extremidades superiores relacionados con el trabajo (Buckle y Devereux, 2002).

Aunque los trastornos musculoesqueléticos que afectan las extremidades inferiores son menos frecuentes que los relacionados con la espalda y las extremidades superiores, los factores de riesgo para los trabajadores en los aserraderos incluyen: posturas prolongadas de pie o sentados, así como trabajo estático y repetitivo. Esto sugiere la necesidad de realizar intervenciones ergonómicas en los puestos de trabajo ya que se observó que los trabajadores frecuentemente realizan sus actividades en esas posiciones durante largos periodos; como es el caso de los operadores de montacargas quienes se ubicaron en el índice global de riesgo más alto con 200 puntos ya que, por la naturaleza de su trabajo, deben permanecer sentados gran parte del tiempo, lo que puede provocar una compresión de las venas y capilares dentro de los músculos, causando falta de apoyo para los pies y microlesiones debido a la ausencia de oxigenación que desencadenan síntomas de desequilibrio, fatiga, malestar y dolor (Vieira y Kumar, 
2004). Finalmente, las actividades que involucran la movilización de materiales en los aserraderos con las rodillas flexionadas y que superan los límites de carga recomendados por la normatividad laboral, también aumentan el riesgo de trastornos musculares en las piernas (Calvo, 2009).

\section{CONCLUSIONES}

Los trabajadores de la industria del aserrío de la región de El Salto, Durango, México, registraron una frecuencia de posturas normales de $52 \%$ que no representa ningún riesgo de que se presenten lesiones musculoesqueléticas; pero en $48 \%$ restante se deben realizar acciones de corrección postural tendientes a minimizar los riesgos en el corto plazo. El índice global de riesgo se estableció en 166 puntos catalogado como mínimo, considerando que a partir de 250 puntos el nivel de riesgo es alto. Los topadores y volteadores son lo más susceptibles de presentar lesiones al exhibir índices globales de riesgo por encima de 200 puntos, y son precisamente en estos puestos donde se sugiere enfocar la atención para dotar de dispositivos de seguridad a los trabajadores.

En general, la posición prototipo de los trabajadores en los aserraderos es trabajar con la espalda doblada o torcida; de pie, haciendo palanca con una pierna para generar tracción; los brazos situados por debajo del nivel de los hombros y desarrollando sobreesfuerzos superiores a los $20 \mathrm{~kg}$; debido a que se han convertido en transportadores de materiales al no contar con mecanismos de transporte mecánico. Así, 43\% de las posiciones corporales en la región de la espalda y $40 \%$ de las piernas se encuentran en un nivel de riesgo 2 , considerado como poco dañino; en especial, el puesto de operador de montacargas que registró un índice global de riesgo de 200 puntos por permanecer la mayor parte del tiempo en posición sentado; lo que a la larga genera malestares y microlesiones en las piernas; en cuanto a los brazos, $100 \%$ de las frecuencias de las posiciones de los brazos resultaron con un nivel de riesgo 1, que no representa riesgo alguno para esta parte del cuerpo.

\section{RECONOCIMIENTOS}

Los autores agradecen al Consejo de Ciencia y Tecnología del Estado de Durango (Cocyted) por el financiamiento al proyecto de investigación titulado: "Evaluación de las condiciones ambientales y riesgos en los puestos de trabajo de la industria forestal de El Salto, Durango" del cual se originó el presente escrito.

\section{REFERENCIAS}

Bello, S. R., \& Mijinyawa, Y. (2010). Assessment of injuries in small scale sawmill industry of south western Nigeria. Agricultural Engineering International: CIGR Journal, 12(1), 151-157. Recuperado de http://www.cigrjournal.org/index.php/Ejounral/article/view/1 $558 / 1293$

Buckle, P. W., \& Devereux, J. J. (2002). The nature of work-related neck and upper limb musculoskeletal disorders. Applied Ergonomics, 33(3), 207-217. doi: 10.1016/S0003-6870(02)00014-5

Calvo, A. (2009). Musculoskeletal disorders (MSD) risks in forestry: a case study to propose an analysis method. Agricultural Engineering International: CIGR Journal, 12(1), 01-09. Recuperado de http://www.cigriournal.org/index.php/Ejounral/article/view/1 $\underline{149 / 1188}$

Chandra, A. M., Ghosh, S., Asahara, S., Barman, S., Dev, S., \& Gangopadhyay, S. (2011). An ergonomic study on musculoskeletal health hazards among sawmill workers of West Bengal, India. Journal of Human Ergology, 40(1-2), 1-78. doi: $\underline{10.11183 / \text { ihe. } 40.1}$

Cheța, M., Marcu, M. V., \& Borz, S. A. (2018). Workload, exposure to noise, and risk of musculoskeletal disorders: A case study of motor-manual tree feeling and processing in poplar clear cuts. Forests, 9(300), 2-20. doi: 10.3390/f9060300

Eroglu, H., Kayacan, Y., \& Yilmaz, R. (2015). Effects of work types and workload on certain anthropometric parameters in forestry workers. The Anthropologist, 20(3), 515-522. doi: $\underline{10.1080 / 09720073.2015 .11891756}$

Gómez-Galán, M., Pérez-Alonso, J., Callejón-Ferre, A. J., \& LópezMartínez, J. (2017). Musculoskeletal disorders: OWAS review. Industrial Health, 55(4), 314-337. doi: 10.2486/indhealth.2016$\underline{0191}$

Gupta, A. (2006). Industrial safety and environment. New Delhi: Laxmi Publications.

Hoogendoorn, W. E., Bongers, P. M., De Vet, H. C. W., Ariens, G. A. M., Van Mechelen, W., \& Bouter, L. M. (2002). High physical work load and low job satisfaction increase the risk of sickness 
absence due to low back pain: results of a prospective cohort study. Occupational and Environmental Medicine, 59(5), 323-328. doi: $\underline{10.1136 / \text { oem. } 59.5 .323}$

IBM Corp. (2010). IBM SPSS Statistics for Windows, Version 19.0. Armonk, NY: IBM Corp.

Janicak, C. A. (2007). Applied statistics in occupational safety and health (2a ed.). Lanham, Maryland: The Scarecrow Press, Inc.

Jones, T., \& Kumar, S. (2010). Comparison of ergonomic risk assessment output in four sawmill jobs. International Journal of Occupational Safety and Ergonomics, 16(1), 105-111. doi: $\underline{10.1080 / 10803548.2010 .11076834}$

Justavino, F. C., Ramírez, R. J., Pérez, N. M., \& Borz, S. A. (2015). The use of OWAS in forest operations postural assessment: advantages and limitations. Bulletin of the Transilvania University of Brasov. Forestry, Wood Industry, Agricultural Food Engineering. Series II, $8(2)$.

Karhu, O., Kansi, P., \& Kuorinka, I. (1977). Correcting working postures in industry: a practical method for analysis. Applied Ergonomics, 8(4), 199-201. doi: 10.1016/0003-6870(77)90164-8

Lee, T. H., \& Han, C. S. (2013). Analysis of working postures at a construction site using the OWAS method. International Journal of Occupational Safety and Ergonomics, 19(2), 245-250. doi: 10.1080/10803548.2013.11076983.

Liu, J., Martínez, L., Wang, H., Rodríguez, R. M., \& Novozhilov, V. (2010). Computing with words in risk assessment. International Journal of Computational Intelligence Systems, 3(4), 396-419. doi: $\underline{10.1080 / 10803548.2013 .11076983}$

Louhevaara, V., Suurnäkki, T., Hinkkanen, S., \& Helminen, P. (1992). OWAS: a method for the evaluation of postural load during work. Helsinki: Institute of Occupational Health. Centre for Occupational Safety.

Consejo Interterritorial del Sistema Nacional de Salud (CISNS). (2000). Protocolos de vigilancia sanitaria específica. Posturas forzadas. Comisión de Salud Pública. Madrid, España. Ministerio de Sanidad, Consumo y Bienestar Social. Recuperado de https://www.mscbs.gob.es/ciudadanos/saludAmbLaboral/docs Lposturas.pdf.

Özkaya, K., Polat, O., \& Kalinkara, V. (2018). Physical workload assessment of furniture industry workers by using OWAS method. The Ergonomics Open Journal, 11,11-19. doi: $\underline{10.2174 / 1875934301811010011}$

Padula, R. S., \& Coury, H. J. C. G. (2003). Sagittal trunk movements during load carrying activities: a pilot study. International Journal of Industrial Eergonomics, 32(3), 181-188. doi: 10.1016/S01698141(03)00062-3
Pascual, S. A., \& Naqvi, S. (2008). An investigation of ergonomics analysis tools used in industry in the identification of work-related musculoskeletal disorders. International Journal of Occupational Safety and Ergonomics, 14(2), 237-245. doi: $\underline{10.1080 / 10803548.2008 .11076755}$

Polat, O., Özkaya, K., \& Kalinkara, V. (2016). Physical workload assessment of workers in furniture industry using the OWAS method. En Second International Furniture Congress, Mugla, Turkey.

Quemelo, P. R. V., Gasparato, F. D. S., \& Vieira, E. R. (2015). Prevalence, risks and severity of musculoskeletal disorder symptoms among administrative employees of a Brazilian company. Work, 52(3), 533-540. doi: 10.3233/WOR-152131

Qutubuddin, S. M., Hebbal, S. S., \& Kumar, A. C. S. (2013). An ergonomic study of work-related musculoskeletal disorder risks in Indian sawmills. Journal of Mechanical and Civil Engineering, 7(5), 713. doi: 10.9790/1684-0750713

RNI Group. (2018). HSE.Ergo.OWAS. Rajawali Nusantara Indonesia Holding Company.

Roman-Liu, D. (2014). Comparison of concepts in easy-to-use methods for MSD risk assessment. Applied Ergonomics, 45(3), 420-427. doi: 10.1016/i.apergo.2013.05.010

Secretaría del Trabajo y Previsión Social [STyPS]. (2014). Norma Oficial Mexicana NOM-006-STPS-2014, Manejo y almacenamiento de materiales-Condiciones de seguridad y salud en el trabajo. Diario Oficial de la Federación. Recuperado de http://www.stps.gob.mx/bp/secciones/dgsst/normatividad/no rmas/Nom-006.pdf

Sharpe, D. (2015). Your chi-square test is statistically significant: Now what?. Practical Assessment, Research \& Evaluation, 20(8), 1-10. Recuperado de https: / pareonline.net/getvn.asp?v $=20 \& n=8$

Spinelli, R., Aminti, G., Magagnotti, N., \& De Francesco, F. (2018). Postural risk assessment of small-scale debarkers for wooden postproduction. Forests, 9(111), 2-12 doi: 10.3390/f9030111

Takala, E. P., Pehkonen, I., Forsman, M., Hansson, G. A., Mathiassen, S. E., Neumann, W. P., ..., \& Winkel, J. (2010). Systematic evaluation of observational methods assessing biomechanical exposures at work. Scandinavian Journal of Work, Environment \& Health, 36(1), 3-24. doi:10.5271/sjweh.2876

Unver-Okan, S., Acar, H. H., \& Kaya, A. (2017). Determination of work postures with different ergonomic risk assessment methods in forest nurseries. Fresenius Environmental Bulletin, 26(12), 7362-7371.

Vieira, E. R., \& Kumar, S. (2004). Working postures: a literature review. Journal of Occupational Rebabilitation, 14(2), 143-159. doi: 10.1023/B:JOOR.0000018330.46029.05 
Zanuttini, R., Cielo, P., \& Poncino, D. (2005). Il metodo OWAS. Prime applicazioni nella valutazione del rischio di patologie muscoloscheletriche nel settore forestale in Italia. Forest@-Journal of Silviculture and Forest Ecology, 2(2), 242. doi: 10.3832/efor02940020242

Manuscrito recibido el 2 de septiembre de 2018

Aceptado el 30 de marzo de 2019

Publicado el 13 de diciembre de 2019
Este documento se debe citar como:

Aragón-Vásquez, A. Y., Silva-Lugo, E. D., Nájera-Luna, J. A., Méndez-González, J., Hernández, F. J., \& de la Cruz-Carrera, R. (2019). Análisis postural del trabajador forestal en aserraderos de El Salto, Durango, México. Madera y Bosques, 25(3), e2531904. doi: 10.21829/myb.2019.2531904

Madera y Bosques por Instituto de Ecología, A.C. se distribuye bajo una Licencia Creative Commons Atribución-NoComercialCompartirlgual 4.0 Internacional. 